\title{
Research on Trajectory Planning in Thunderstorm Weather Based on Dynamic Window Algorithm during Approach Segment
}

\author{
Li Lu (iD) and Chenyu Liu \\ School of Air Traffic Management, Civil Aviation Flight University of China, Sichuan, Guanghan 618307, China \\ Correspondence should be addressed to Li Lu; 421361599@qq.com
}

Received 29 June 2021; Accepted 15 December 2021; Published 10 January 2022

Academic Editor: Pengwei Wang

Copyright (C) $2022 \mathrm{Li} \mathrm{Lu}$ and Chenyu Liu. This is an open access article distributed under the Creative Commons Attribution License, which permits unrestricted use, distribution, and reproduction in any medium, provided the original work is properly cited.

\begin{abstract}
Dynamic window algorithm (DWA) is a local path-planning algorithm, which can be used for obstacle avoidance through speed selection and obtain the optimal path, but the algorithm mainly plans the path for fixed obstacles. Based on DWA algorithm, this paper proposes an improved DWA algorithm based on space-time correlation, namely, space-time dynamic window approach. In SDWA algorithm, a DWA associated with obstacle position and time is proposed to achieve the purpose of path planning for moving obstacles. Then, by setting the coordinates of the initial moving obstacle and identifying safety distance, we can define the shape of the obstacle and the path planning of the approach segment in thunderstorm weather based on the SDWA model was realized. Finally, the superior performance of the model was verified by setting moving obstacles for path planning and selecting the aircraft approach segment in actual thunderstorm weather. The results showed that SDWA has good path-planning performance in a dynamic environment. Its path-planning results were very similar to an actual aircraft performing thunderstormavoidance maneuvers, but with more smooth and economical trajectory. The proposed SDWA model had great decision-making potential for approach segment planning in thunderstorm weather.
\end{abstract}

\section{Introduction}

To ensure the flight safety of the aircraft, the path planning of the approach segment in thunderstorm weather is mainly to reasonably design the diversion route for aircrafts that may encounter thunderstorms on future route within a defined period of time. During approach, the aircraft's altitude decreases and is susceptible to thunderstorm hazards [1-5]. Thunderstorms produce violent updrafts and downdrafts; flying into it will cause serious damage to the aircraft. There is a large amount of super cooled water in the thunderstorm, which can result in icing on the exterior and thus hinders aircraft landing. Meanwhile, air collision will produce electric shock that can damage the protruding parts of the fuselage and electronic equipment $[6,7]$. Therefore, how to effectively plan the path in thunderstorm weather is of great significance $[8,9]$.

Path planning is widely used in engineering. In the past few decades, a variety of path-planning models have been proposed according to different angles. Path planning for fixed obstacles includes intelligent algorithm path planning, such as ant colony algorithm [10-13], traditional pathplanning algorithms, such as artificial potential field method [14-16], and hybrid path-planning algorithms, such as potential field ant colony fusion method [17], Genetic fuzzy algorithm [18], and fuzzy neural network algorithm [19]. Path planning of moving obstacles includes Jiang Zhongyu's "Improved dynamic a $\times$ algorithm combined with time window" [20], Wu Chuanjun's "moving obstacle path planning based on dynamic spatiotemporal environment impact model and grid" [21], and Chen Jinfeng's "artificial potential field method combined with rolling window" [22]. For the approach segment path planning in civil aviation thunderstorm weather, the traditional algorithm $[23,24]$ is mainly used to plan the path of aircraft with thunderstorm on the route.

The dynamic window approach is a path-planning algorithm that drives objects by simulating trajectories at 
different speeds, evaluating the trajectories, and selecting the speed under the optimal trajectory in the current window [25]. It can deal with a series of obstacle avoidance problems of fixed obstacles. In recent years, people have proposed a series of variants of DWA algorithm, such as DWA algorithm [26-28] combined with a $\times$ algorithm, which has good application effects in solving path-planning problems in complex environments.

Due to the fixed height of the aircraft in the approach segment, a problem concerning the diversion path of the approach segment in thunderstorm weather is in fact a pathplanning problem. However, thunderstorms are often dynamically unpredictable; using the traditional DWA algorithm is merely insufficient. Therefore, this paper designs a Dynamic Window Algorithm (SDWA) for path planning of moving obstacles through polynomial fitting of obstacle positioning and time in a single window, so as to efficiently and timely plan the diversion path in advance for thunderstorms that will appear on the route. As an example, the effectiveness of the algorithm is verified by taking the diversion of approach segment under actual thunderstorm weather at Taipei Taoyuan Airport.

\section{The Establishment and Simulation of the Model}

2.1. Establishment of SDWA. SDWA algorithm mainly includes the establishment of aircraft motion model and dynamic obstacle environment. The main process is shown in Figure 1.

\subsubsection{Establishment of the Aircraft Motion Model}

(1) Establishment of Aircraft Motion Equation. Due to the limitation of flight procedure, the aircraft cannot escape the protected area of the flight route. However, in the departure and arrival segments, the flight altitude is relatively low and can be easily affected by thunderstorm weather, forcing the aircraft to reroute. Therefore, this paper focuses on how to obtain the predicted flight trajectory before an aircraft encountering a thunderstorm.

In the approach segment, the aircraft has strict requirements on the altitude of rise and descend adjustments. Therefore, the diversion measures for thunderstorm clouds in the approach segment are mainly fly-around-motion [29]:

$$
V_{t}=\left(v_{t}, w_{t}\right) \text {, }
$$

where $v_{t}$ is the linear velocity of the aircraft at time $t$ and $w_{t}$ is the angular velocity of the aircraft. The aircraft trajectory is described, as shown in Figure 2:

When the distance between two adjacent moments is small enough, the trajectory can be regarded as a straightline flight:

$$
\left\{\begin{array}{l}
\Delta x=v_{t} \Delta t \cos \left(\alpha_{t}\right) \\
\Delta y=v_{t} \Delta t \sin \left(\alpha_{t}\right)
\end{array}\right.
$$

Therefore, the coordinates of the aircraft at a certain time (i.e., the accumulation of the previous displacements) and the yaw angle of the aircraft can be expressed as

$$
\left\{\begin{array}{l}
x_{t+1}=x_{t}+v_{t} \Delta t \cos \left(\alpha_{t}\right) \\
y_{t+1}=y+v_{t} \Delta t \sin \left(\alpha_{t}\right) \\
\alpha_{t+1}=\alpha_{t}+w_{t} \Delta t
\end{array}\right.
$$

(2) Aircraft Motion parameters. There are infinite groups of velocities in two-dimensional space; due to the influence of aircraft itself and the thunderstorm, the velocity can be constrained by the following restrictions:

(1) Speed limit under self-performance guarantee:

$$
v_{t}=\left\{v \in\left[v_{\min }, v_{\max }\right], w \in\left[w_{\min }, w_{\max }\right]\right\} .
$$

(2) Aircraft is limited by its own engine performance:

$$
V_{t}\left\{(v, w) \mid \begin{array}{c}
v \in\left[v_{t-1}-v_{a} \Delta t, v_{t-1}+v_{b} \Delta t\right] \\
\cap w \in\left[w_{t-1}-w_{a} \Delta t, w_{t-1}+w_{b} \Delta t\right]
\end{array}\right\},
$$

where $v_{a}$ and $w_{a}$ are the maximum braking linear acceleration and angular acceleration that the aircraft can provide at $t-1$ and $v_{b}$ and $w_{b}$ are the maximum driving linear acceleration and angular acceleration of the aircraft at $t-1$.

(3) Based on the distance limit between the aircraft and the thunderstorm.

In order to avoid the thunderstorm during the approach segment, the aircraft will decelerate with the maximum braking acceleration:

$$
\left\{\begin{array}{l}
V_{t}^{2}=\left\{\left(v^{2}, w^{2}\right)\left[\begin{array}{c}
v^{2} \leq 2 v_{a} D\left(v_{t-1}, w_{t-1}\right) \\
\cap w^{2} \leq 2 w_{a} D\left(v_{t-1}, w_{t-1}\right)
\end{array}\right]\right\}, \\
V_{t} \geq 0
\end{array}\right.
$$

where $D$ is the minimum distance between the aircraft and the thunderstorm at $t-1$.

(3) Aircraft speed evaluation function.

(1) Establishment of evaluation function index:the speed evaluation index of aircraft is mainly determined by three indexes, as shown in Table 1.

(2) Normalization of indicators: since the three indexes are of different dimensions, they are normalized [30] as follows:

$$
x(i)=\frac{x(i)}{\sum_{i=1}^{k} x(i)} \text {. }
$$

$x(i)$ is the $x$ index of $i$ trajectories in the current dynamic window algorithm [31] and $k$ is all trajectories in the current dynamic window.

(3) Speed evaluation function.

Based on the existing evaluation index, the proportion is set to establish a comprehensive evaluation function: 


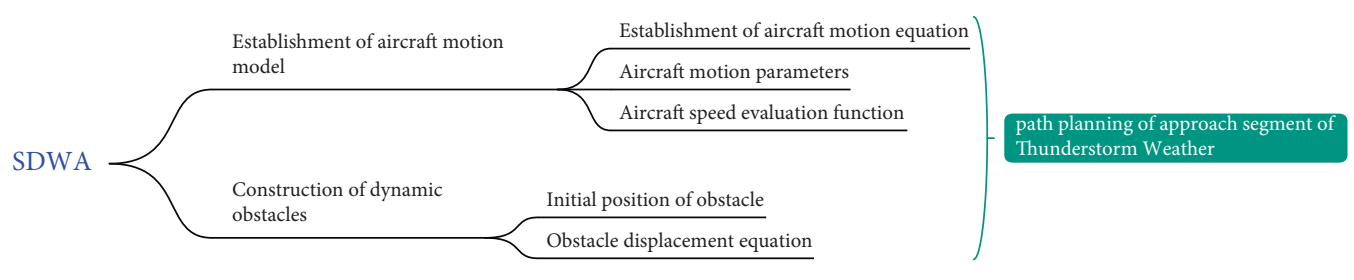

Figure 1: The flow of SDWA.

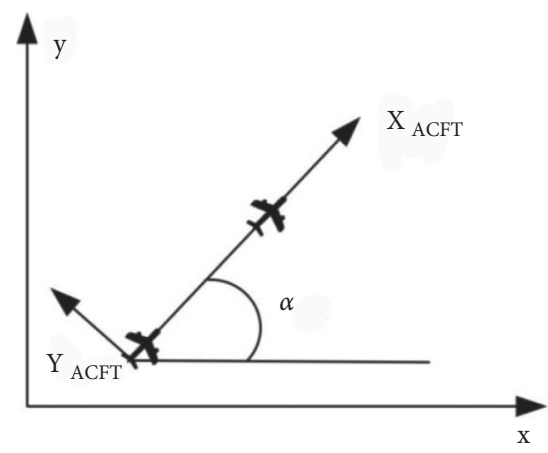

Figure 2: Aircraft flight model diagram.

TABLE 1: Flight speed evaluation index table.

Heading angle

Distance from thunderstorm

$A(v, w)=\pi-\theta(\theta$ is the angle between the current course of the aircraft and the target direction)

Speed

$D(v, w)$

$V(v, w)$

$$
H(v, w)=\beta A+\gamma D+\varepsilon V .
$$

Among $\beta, \gamma$, and $\varepsilon$, in this paper, the safety distance from thunderstorm cloud is the main evaluation index $\beta=0.3, \gamma=0.4$, and $\varepsilon=0.3$.

\subsubsection{Setting of Dynamic Obstacles}

(1) Setting of Initial Position of Obstacle. When the aircraft arrives at the approach segment, the location of thunderstorm can be described as follows:

$$
P=(x, y) \text {. }
$$

(2) Obstacle Moving Trajectory Equation. After the aircraft arrives at the approach segment, the moving trajectory of obstacles can be described by establishing the trajectory equation related to $t$, and the trajectory equation is as follows:

$$
\left\{\begin{array}{l}
x=f(t), \\
y=g(t) .
\end{array}\right.
$$

2.2. Matlab Model Simulation. According to the established aircraft motion model, Matlab is used for simulation [32] to observe whether the predicted route of the aircraft to avoid thunderstorm is reasonable. Here, the shape of thunderstorm is set as a round thunderstorm with a circular trajectory (that is, moving obstacles are composed of four adjacent points in the simulation process). The specific algorithm flow is shown in Figure 3, and the parameters are shown in Table 2.

The simulation results are shown in Figure 4.

It can be seen from Figure 4 that the thunderstorm moves in a circular area with $(2,2.5)$ as the center and $50 \mathrm{~km}$ as the radius. The aircraft flies at the initial heading angle $\pi$ 4. The model was successfully used to avoid the moving thunderstorm, which verified the feasibility of the model and compared with other path-planning algorithms in dynamic environment; this method makes use of the dynamic characteristics of DWA, and the algorithm has fast solution speed and high efficiency.

\section{Flight Example Verification}

To verify the reliability of the model, this paper takes the BR721 flight on PVG-TPE of TPE approach segment on June 6, 2021, as an example, as shown in Figure 5.

In the picture, the weather radar echo over TPE is red, and flight BR721 will encounter thunderstorm according to the original planned route over TPE. Therefore, the timedependent displacement parameter equation can be generated by predicting the thunderstorm trajectory, which can be brought into the DWA model of this paper for collision prediction and rerouting planning. 


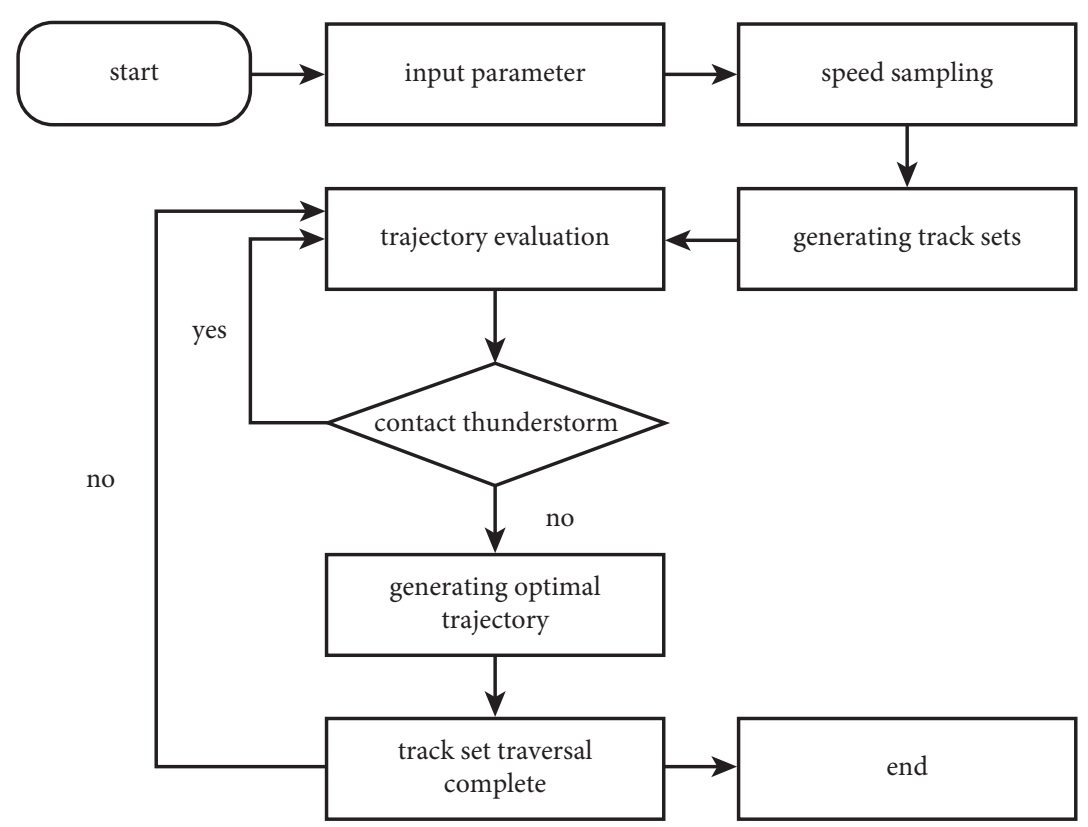

Figure 3: Dynamic window algorithm flowchart.

TABLE 2: Initial simulation parameter table.

\begin{tabular}{lc}
\hline Aircraft initial position $(50 \mathrm{~km})$ & $(0,0)$ \\
\hline Initial yaw angle of aircraft $(\mathrm{rad})$ & $\pi / 4$ \\
Aircraft initial speed $(\mathrm{km} / \mathrm{s})$ & $V=0$ \\
Target location $(50 \mathrm{~km})$ & $(9,9)$ \\
Maximum speed $(\mathrm{km} / \mathrm{h})$ & 400 \\
Maximum yaw speed $(\mathrm{rad} / \mathrm{s})$ & 20.0 \\
Acceleration $(\mathrm{km} / \mathrm{ss})$ & 0.001 \\
Rotational acceleration $(\mathrm{rad} / \mathrm{ss})$ & 1 \\
Thunderstorm track $(\mathrm{km})$ & $(y=\cos t+2.5, x=\sin t+1)$ \\
Thunderstorm shape $(50 \mathrm{~km})$ & $(x, y)(x+1, y)(x, y+1)(x+1, y+1)$ \\
Minimum distance from thunderstorm cloud $(50 \mathrm{~km})$ & 0.25 \\
\hline
\end{tabular}

\subsection{Establishing Thunderstorm Displacement Parameter} Equation. Through the observation of weather radar echo map over TPE at different times (as shown in Figure 6), thunderstorms can be found moving to the east over the northwest of TPE. The thunderstorm displacement images displayed by weather radar echo within 210 minutes are superimposed by graphic software, and the thunderstorm displacement trajectory is shown in Figure 7.

To fit the thunderstorm trajectory equation, taking TPE as the starting point, the direction to the east as the $X$-axis, and the direction to the north as the $Y$-axis, a rectangular coordinate system is established to obtain the thunderstorm displacement model, as shown in Figure 8 .

The thunderstorm trajectory can be found as a curve through the model diagram. Therefore, the parameter equation of thunderstorm trajectory can be obtained by polynomial regression fitting. The thunderstorm coordinates at each time are shown in Table 3:

The above data were fitted by polynomial regression [33], and the fitting formula was as follows:

$$
\left\{\begin{array}{l}
x=a_{n} t^{n}+a_{n-1} t^{n-1}+\cdots+a_{1} t+a_{0}, \\
y=a_{n} t^{n}+a_{n-1} t^{n-1}+\cdots+a_{1} t+a_{0} .
\end{array}\right.
$$

The fitting results are shown in Figures 9 and 10 .

Through the comparison of fitting results, to avoid overfitting, the $x$ and $y$ coordinates are fitted by third-order polynomial as the parameter equation of thunderstorm displacement trajectory; the parameter equation is as follows:

$\left\{\begin{array}{l}x=-0.000005 t^{3}-0.0028 t^{2}+2.0297 t-154.3332 \\ y=0.00002 t^{3}-0.0082 t^{2}+1.0176 t+48.7011\end{array}\right.$

3.2. Simulation. The parameters [34], thunderstorm shape, and displacement equation are brought into the model and simulated by MATLAB. The simulation data are shown in Table 4.

The simulated thunderstorm position and aircraft position are shown in Table 5, and the results are shown in Figure 11: 

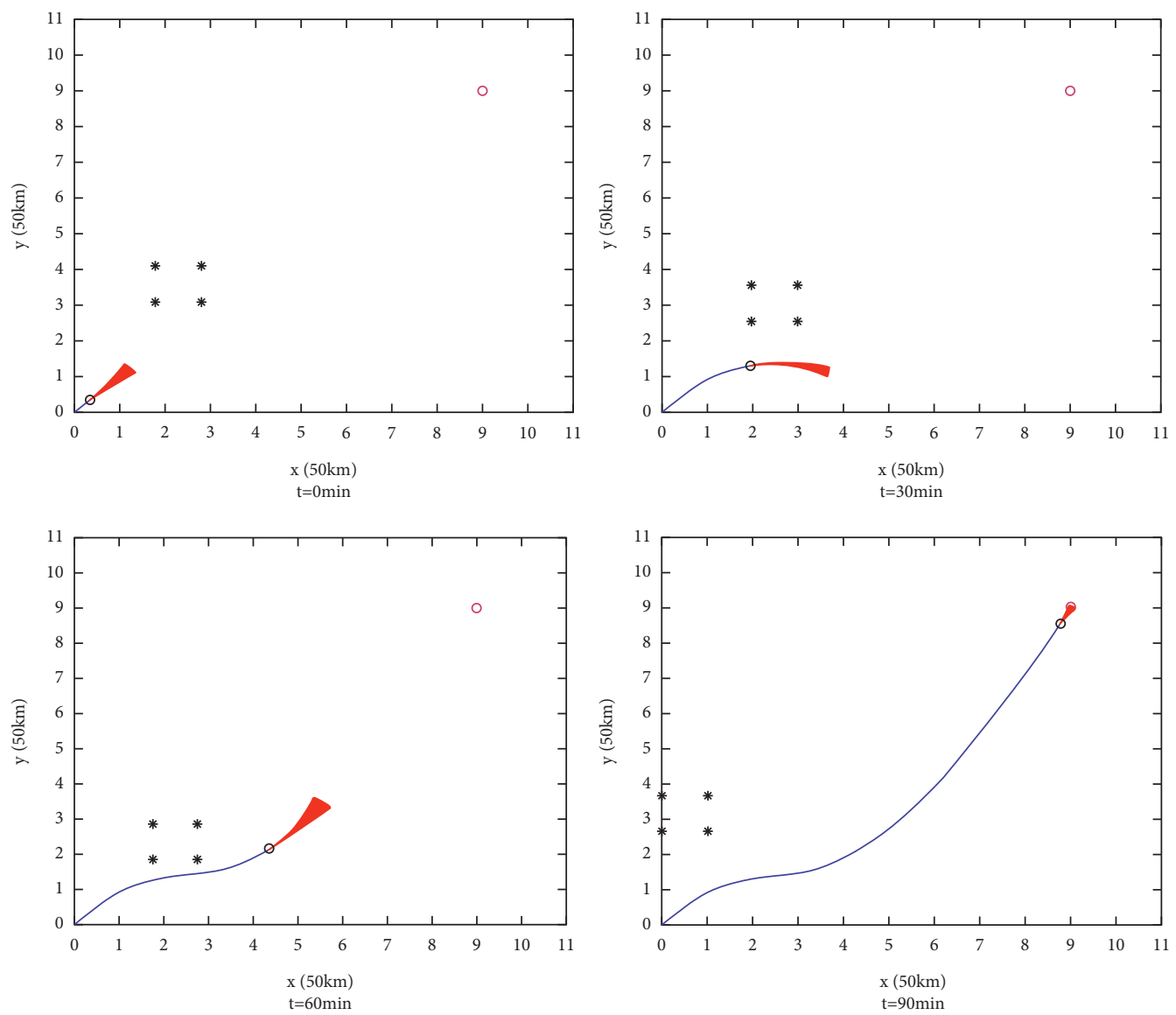

FIGURE 4: Simulation of flight diversion route for aircraft avoiding thunderstorm.

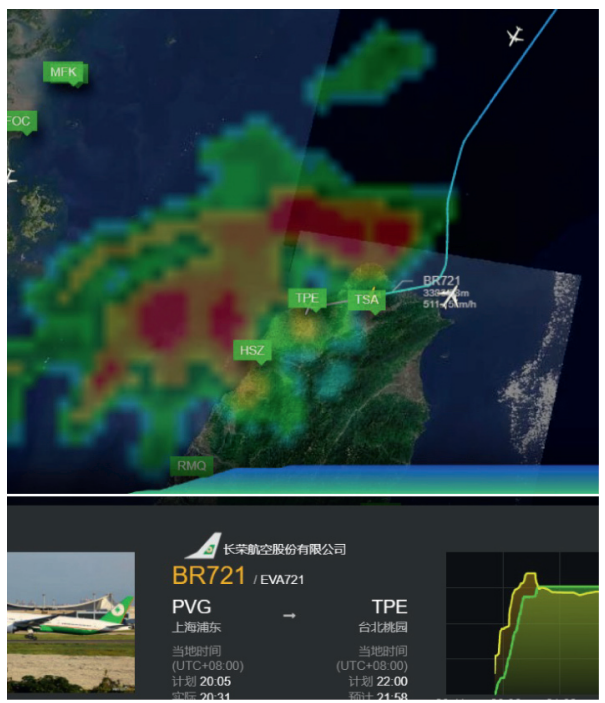

Figure 5: Flight chart of BR721. 


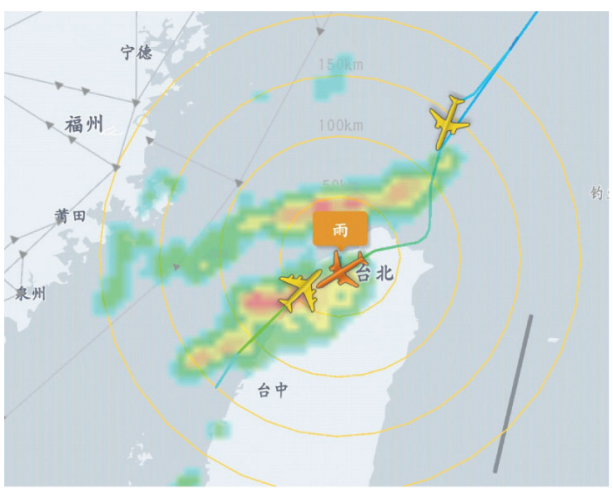

$\mathrm{t}=0 \mathrm{~min}$

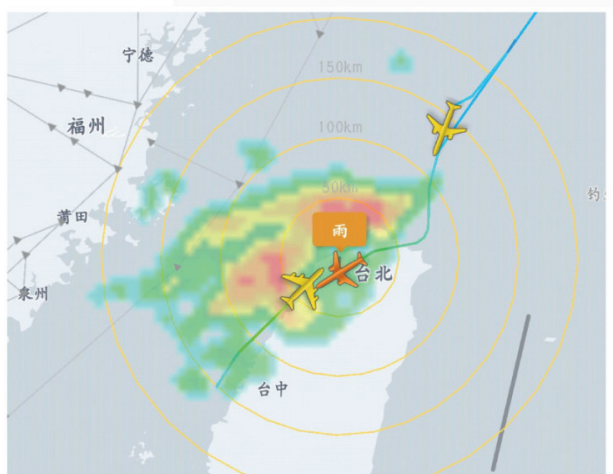

$\mathrm{t}=140 \mathrm{~min}$

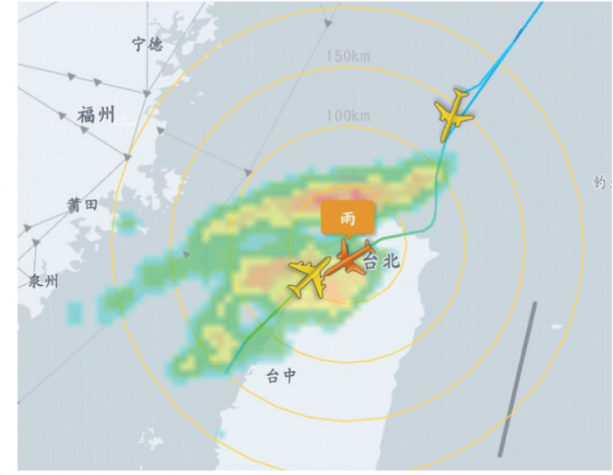

$\mathrm{t}=70 \mathrm{~min}$

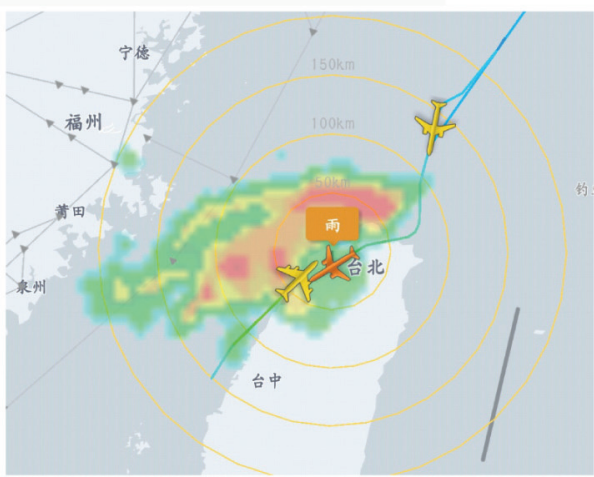

$t=210 \mathrm{~min}$

FIGURE 6: Location map of thunderstorm at each time point.

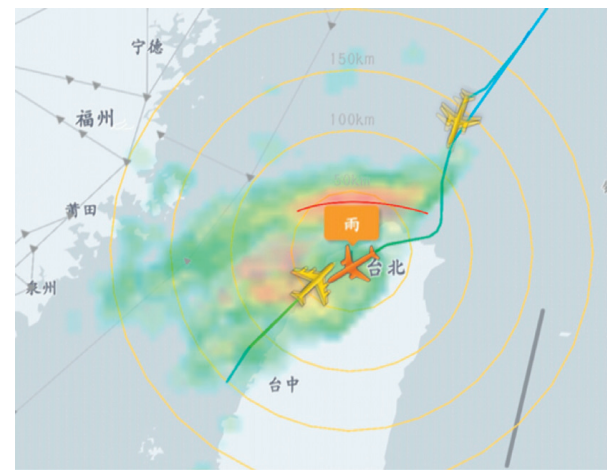

Figure 7: Thunderstorm trajectory.

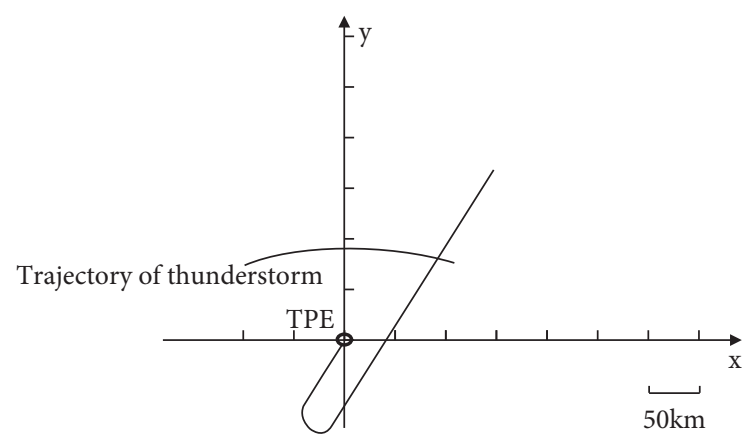

Figure 8: Flight movement model diagram. 
TABle 3: Thunderstorm time table.

\begin{tabular}{lcccccc}
\hline$t(\min )$ & 35 & 70 & 95 & 130 & 165 & \\
\hline$x(\mathrm{~km})$ & -86.4 & -30.3 & 10.6 & 51.2 & 80.4 & 102.3 \\
$y(\mathrm{~km})$ & 75.3 & 85.4 & 87.7 & 83.2 & 75.2 & 70.2 \\
\hline
\end{tabular}
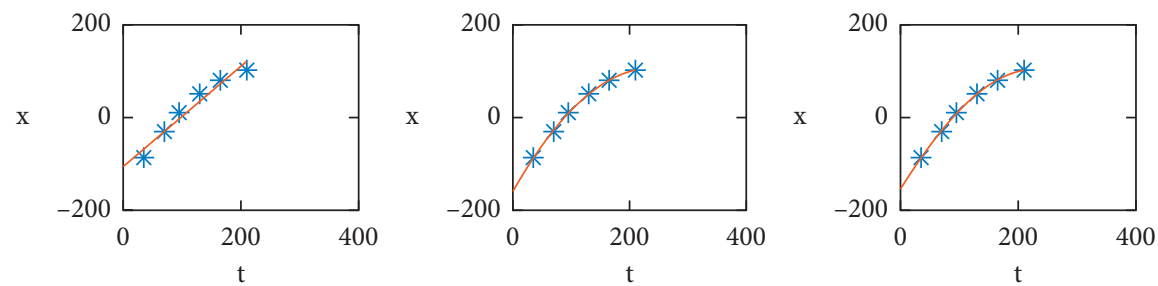

Figure 9: Thunderstorm $x$-coordinate $1-3$ order fitting diagram.
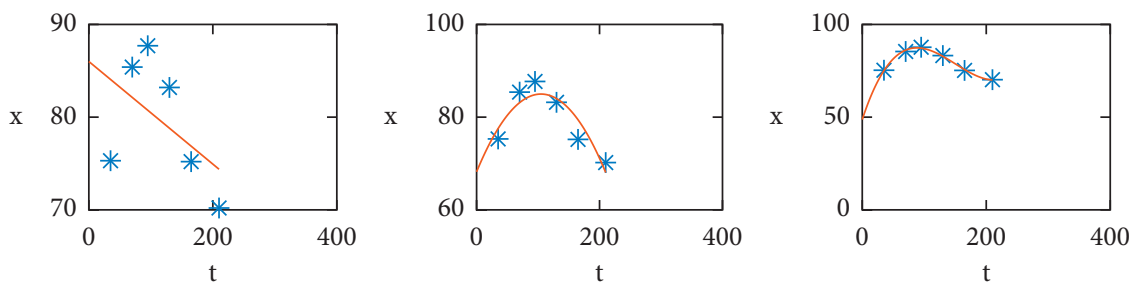

FIGURE 10: Thunderstorm $y$-coordinate $1-3$ order fitting diagram.

TABLE 4: Case simulation parameters.

\begin{tabular}{lc}
\hline Aircraft initial position $(\mathrm{km})$ & $(300,300)$ \\
\hline Initial yaw angle of aircraft $(\mathrm{rad})$ & $-\pi / 4$ \\
Aircraft initial speed $(\mathrm{km} / \mathrm{h})$ & 800 \\
Starting position of approach $(\mathrm{km})$ & $(-10,-30)$ \\
Runway entrance location $(\mathrm{km})$ & $(0,0)$ \\
Maximum speed $(\mathrm{km} / \mathrm{h})$ & 800 \\
Maximum yaw speed $(\mathrm{rad} / \mathrm{s})$ & 20.0 \\
Acceleration $(\mathrm{km} / \mathrm{ss})$ & 0.01 \\
Rotational acceleration $(\mathrm{rad} / \mathrm{ss})$ & 1 \\
Thunderstorm track $(\mathrm{km})$ & $x=-0.000005 t^{3}-0.0028 t^{2}+2.0297 t-154.3332 y=0.00002 t^{3}-0.0082 t^{2}+1.0176 t+48.7011$ \\
Thunderstorm shape $(\mathrm{km})$ & $(x, y) 、(x-50, y+25) 、(x-50, y) 、(x-100, y)(x, y-20)$ \\
$\begin{array}{l}\text { Minimum distance from thunderstorm } \\
\text { cloud }(\mathrm{km})\end{array}$ & 25 \\
\hline
\end{tabular}

TABlE 5: The position of thunderstorm simulation and aircraft parameters.

\begin{tabular}{lcccccc}
\hline$t(\min )$ & 35 & 70 & 95 & 130 & 165 & \\
\hline$X_{\text {clo }}(\mathrm{km})$ & -86.4 & -30.3 & 10.6 & 51.2 & 80.4 & 102.3 \\
$Y_{\text {clo }}(\mathrm{km})$ & 75.3 & 85.4 & 87.7 & 83.2 & 75.2 & 0.2 \\
$X_{\text {air }}(\mathrm{km})$ & 290.7 & 220.3 & 120.1 & 100.3 & 0 \\
$Y_{\text {air }}(\mathrm{km})$ & 292.1 & 224.1 & 115.7 & 12.3 & -25.3 & 0 \\
\hline
\end{tabular}

It can be seen from Figure 11 that the aircraft successfully avoided the moving thunderstorm from the beginning of approach to the end, and its simulated track (blue) is smoother and more operable and economical than the actual track (red) [35], which provides a new and effective method for approach segment path planning in thunderstorm weather. 

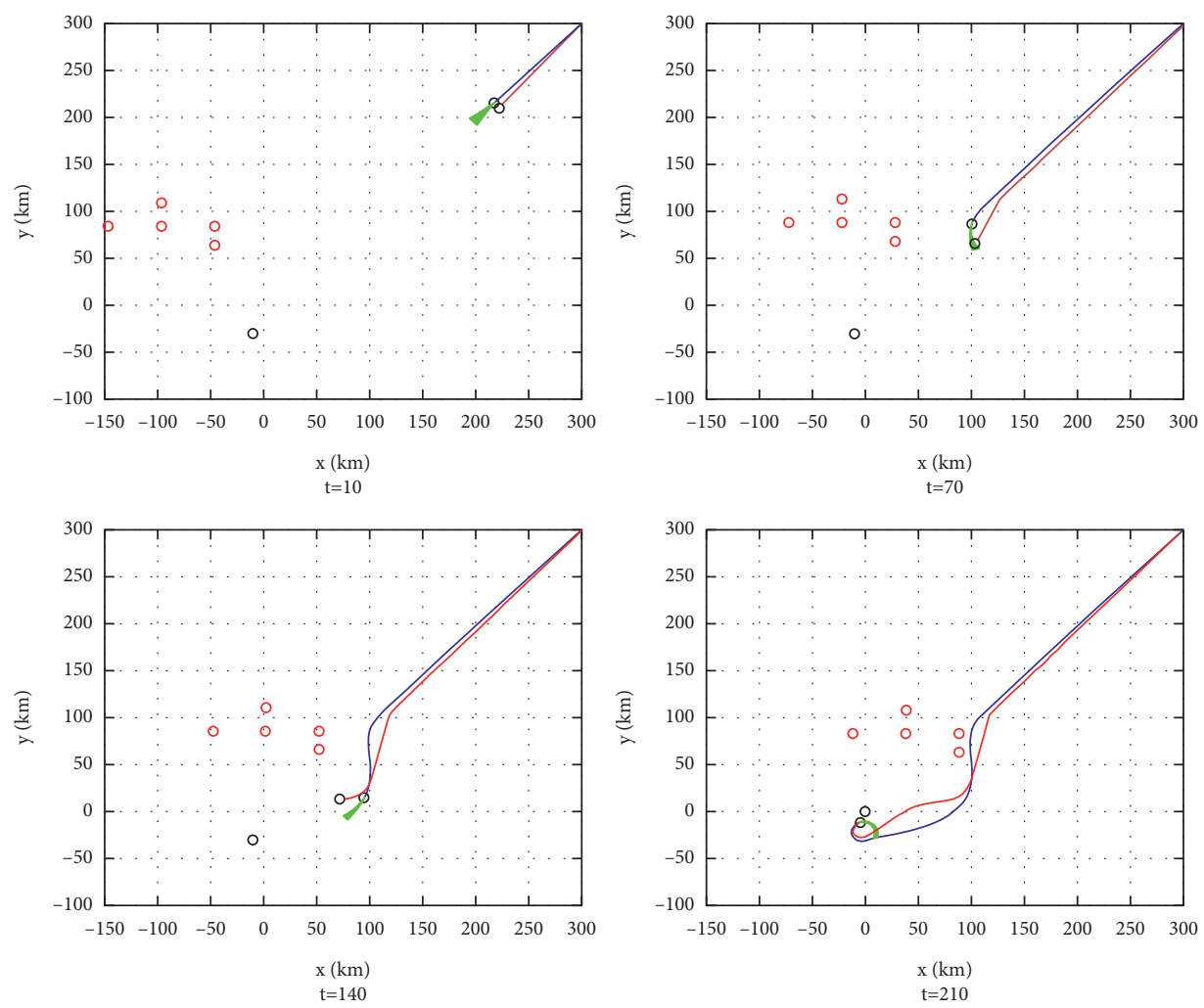

FIGURE 11: Dynamic simulation of flight BR721 avoiding thunderstorm.

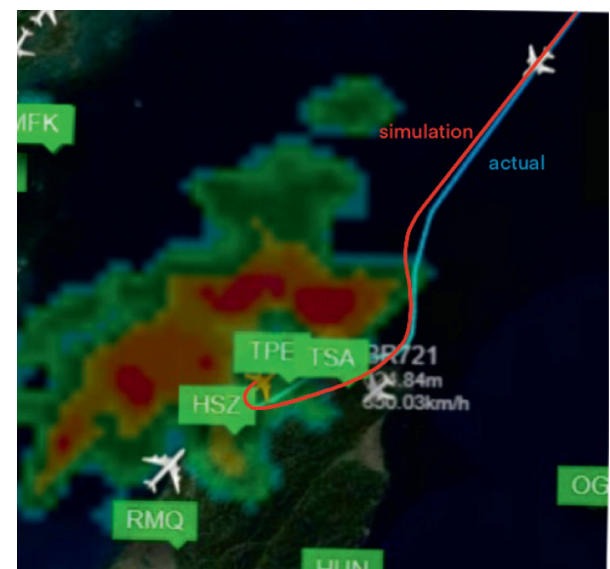

FIGURE 12: Comparison chart of the simulated route and the actual route.

\section{Conclusion}

In this paper, an improved spatiotemporal dynamic window approach is proposed. The algorithm can deal with the pathplanning problem in an dynamic environment by associating the location of obstacles in a single window with time. In SDWA, the traditional DWA algorithm is used for fixed obstacle avoidance along the path, and the location of obstacles in each window is refreshed by time-related location function. Next, move the obstacles under the continuous window along/on with the target trajectory to complete the construction of the dynamic environment. Meanwhile, to solve the trajectory-planning problem of the approach segment in thunderstorm weather, aiming at the complex shape of thunderstorm, SDWA describes the thunderstorm shape through the setting of obstacle point set, sets relevant motion parameters with reference to the performance of relevant civil aircraft, and sets parameters such as safety distance in combination with relevant requirements for safe flight of civil aircraft. Through the regression analysis of thunderstorm displacement trajectory and time, the thunderstorm displacement trajectory equation is established and applied to constructing a dynamic environment. Finally, SDWA is used to plan the trajectory of the approach segment in thunderstorm weather. The simulated diversion track and the actual diversion track are shown in Figure 12 (blue is the actual motion track and red is the simulation track).

The results show that the SDWA algorithm has good obstacle avoidance performance, which makes reasonable trajectory planning for the aircraft with thunderstorm weather on the route and has highly application to the actual circumstance, and the trajectory is smoother and more economical. Therefore, SDWA provides a safe and efficient method to solve the problem of approach segment path planning under thunderstorm weather. In the future, research should further combine GPS positioning system or Beidou navigation system to improve the implementation accuracy of simulation route [36-38]. Under the new route system, it can further improve the efficiency and safety of diversion planning in thunderstorm weather [39].

However, when SDWA deals with an extremely complicated weather, it may fall into a local loop of the path due 
to the problem of parameters' setting, resulting in obtaining the optimal path difficultly. Therefore, the model can be further optimized by combining the optimization algorithm in the future.

\section{Data Availability}

All the data in the study are available.

\section{Conflicts of Interest}

The authors declare that there are no conflicts of interest regarding the publication of this paper.

\section{Acknowledgments}

The authors are very grateful for the support of the youth fund by Civil Aviation Flight University of China (Q2020118 ) and construction of advantageous characteristic subject of Transportation Engineering (Central Universities establish the first-class university of the world (subject) in 2021 and the special Project for Guiding Characteristic Development) (D202103).

\section{References}

[1] W. Fan, Research on the Influence of Thunderstorm on Aviation Flight, Chengdu University Of Information Technology, Chengdu, China, 2018.

[2] M. Zhang, "Discussion on the characteristics of civil aviation flight in thunderstorm weather," Electronic testing, no. 15, pp. 165-166, 2015.

[3] X. Huang, "Impact of meteorological conditions on civil aviation flight safety," Science and technology and enterprise, no. 17, p. 180, 2015.

[4] Y. Jia, "Thunderstorm weather and flight safety," Knowledge is power, no. 9, p. 53, 2008.

[5] Y. Su, Z. Pei, and X. Chang, "Impact of climate change on civil aviation operation," Civil aviation management, no. 11, pp. 60-62, 2019.

[6] Y. Nikitin, P. Božek, and J. Peterka, "Logical-linguistic model of diagnostics of electric drives with sensors support," Sensors, vol. 20, no. 16, p. 4429, 2020.

[7] I. V. Abramov, A. I. Abramov, Y. R. Nikitin, E. Sosnovich, P. Bozek, and V. Stollmann, "Diagnostics of electrical drives," in Proceedings of the International Conference on Electrical Drives and Power Electronics (EDPE), pp. 364-367, Tatranska Lomnica, Slovakia, September 2015.

[8] J. Bai, "Thunderstorm weather characteristics and control and command methods in Yinchuan Hedong airport," Civil aviation management, no. 8, pp. 69-71, 2016.

[9] F. Wu, H. Xiao, and Y. Cheng, "Analysis on the supporting role of meteorological services in the whole process decisionmaking of civil aviation transportation industry," Guangdong meteorology, vol. 37, no. 1, pp. 47-51, 2015.

[10] H. Xin and H. Yang, "UAV route planning based on guided ant colony optimization," Microcomputer application, vol. 37, no. 8, pp. 89-92, 2021.

[11] Z. Li, J. Li, P. Wang, and B. Xu, "Research on path planning of intelligent vehicle handshake area based on improved ant colony algorithm," Data communication, no. 4, pp. 47-51, 2021.
[12] F. Yuan and J. Zhu, "Optimal path planning of mobile robot based on improved ant colony algorithm," Modern Manufacturing Engineering, no. 7, pp. 38-47 + 65, 2021.

[13] Y. Li, Y. Ji, and J. Zhao, "Research on vehicle obstacle avoidance system based on ant colony algorithm," Hebei agricultural machinery, no. 8, pp. 82-83, 2021.

[14] y. Deng and X. Jiang, "Trajectory planning algorithm of four rotor UAV Based on improved artificial potential field method," Sensors and Microsystems, vol. 40, no. 7, pp. 130133, 2021

[15] Y. Fan, B. Huang, Q. Zhou, and H. Chen, "Research on obstacle avoidance path planning of trolley based on improved artificial potential field method," Software guide, vol. 20, no. 7, pp. 38-42, 2021.

[16] C. S. Zhang, J. Wang, L. Zhang, and Y. Wang, "Multi agent system cluster obstacle avoidance model for complex obstacle field," Acta Ordnance Engineering Sinica, no. 1, pp. 1-12, 2021, http://kns.cnki.net/kcms/detail/11.2176.TJ.20210104.1603. 012.html.

[17] P. Xiang, F. Xiang, and J. Mao, "Path planning method of mobile robot under multi terrain constraints," Small microcomputer system, vol. 42, no. 9, pp. 1900-1905, 2021.

[18] H. Lu, J. Yan, Y. Xing Bao et al., "Obstacle avoidance simulation of wall climbing robot based on genetic fuzzy algorithm," Light industry machinery, vol. 39, no. 1, pp. 82-85, 2021.

[19] L. Zheng, Research on Obstacle Avoidance Planning Algorithm of Unmanned Ship, Chongqing University of Posts and telecommunications, Chongqing, China, 2020.

[20] Z. Jiang, Research on Path Planning of Multi Handling Robot Under Moving Obstacles, Beijing Jiaotong University, Beijing, China, 2020.

[21] C. Wu, L. Guan, Q. Xia, G. Chen, and Y. Dong, "Shortest time path planning for predictable dynamic spatiotemporal environment," Journal of Surveying and Mapping Science and technology, vol. 38, no. 3, pp. 316-322, 2021.

[22] C. Jin, F. Huang, W. Hua, Z. Zhang, and Q. Li, "Path planning algorithm based on improved artificial potential field method in dynamic environment," Modular machine tool and automatic processing technology, no. 12, pp. 6-9 + 14, 2020.

[23] X. Zheng, W. Zhang, and W. Zhang, "Path planning based on multi aircraft collision avoidance in thunderstorm weather," Chinese Journal of safety Sciences, vol. 29, no. 8, pp. 151-156, 2019.

[24] Z. Lv, Q. Wang, Y. Jin, and T. Yuan, "Research on rerouting model under supercell thunderstorm," Flight mechanics, vol. 35, no. 2, pp. 88-91, 2017.

[25] X. Chang, W. Hu, S. Ji, and Y. Yue, "Research on obstacle avoidance of mobile robot based on improved Dynamic window approach," Modular machine tool and automatic machining technology, no. 7, pp. 33-36, 2021.

[26] Z. Zhang, P. Zhang, H. Mao, X. Li, and Q. Sun, "Global dynamic path planning integrating improved a $*$ algorithm and dynamic window approach," Electro Optic and Control, 2021, http://kns.cnki.net/kcms/detail/41.1227.tn.20210616. 1718.004.html.

[27] J. Chen, C. Jia, and Q. Liu, "Path planning of mobile robot based on improved a $*$ and dynamic window algorithm," Computer integrated manufacturing system, 2021, http://kns. cnki.net/kcms/detail/11.5946.TP.20201026.1053.026.html.

[28] W. Zhao and $\mathrm{Z}$. Wu, "Dynamic path planning of bilevel optimization a * algorithm and Dynamic window approach," Computer Engineering and Application, 2021, http://kns.cnki. net/kcms/detail/11.2127.TP.20210520.1000.002.html. 
[29] X. Liu and H. Yan, "Emergency path planning method for one engine failure based on dynamic window algorithm," Journal of Civil Aviation Flight College of China, vol. 32, no. 1, pp. 17-21, 2021.

[30] X. Ma, Z. Yao, T. Wu, and M. Wang, Data Analysis Method and Application, People's Posts and Telecommunications Press, Beijing, China, 2015.

[31] L. Chang, L. Shan, C. Jiang, and Y. Dai, "Reinforcement based mobile robot path planning with improved dynamic window approach in unknown environment," Autonomous Robots, vol. 45, 2020 (prepublish).

[32] L. Shi, Super Learning Manual of MATLAB/Simulink System Simulation, People's Posts and Telecommunications Press, Beijing, China, 2014.

[33] Z. Xia and H. Liang, "Trajectory planning of palletizing robot based on polynomial fitting interpolation function," Shandong Science, vol. 29, no. 5, pp. 117-123, 2016.

[34] G. He, Li Lu, J. Hu, and X. Ma, "Study on flight diversion decision under thunderstorm weather based on least square method," Safety and Environmental Engineering, vol. 26, no. 4, pp. 171-176, 2019.

[35] Y. Xue, Y. Jing, and G. Fu, "Statistical analysis on the causes and action paths of unsafe actions in general aviation flight accidents," Safety and environmental engineering, vol. 25, no. 2, pp. 131-213, 2018.

[36] Q. Wen, "Application of GPS positioning system and Beidou navigation system in civil aviation," Scientific and technological innovation and application, no. 28, pp. 168-169, 2019.

[37] S. Fan, L. pan, and Y. Ma, "Civil aviation technology application system based on Beidou navigation," Journal of civil aviation, vol. 5, no. 1, pp. 9-15, 2021.

[38] W. Pan, Research on Real-Time Airborne Araim for Civil Aviation Precision Approach, Shanghai Jiaotong University, Shanghai, China, 2020.

[39] Z. Fu, "Research and Analysis on integrated operation of air traffic control and meteorological information," in Proceedings of the 1st Annual Academic Conference on air traffic management system technology, vol. 8, Naples, Italy, May 2018. 